\title{
The Role of Screening in Depression Diagnosis and Treatment in a Representative Sample of US Primary Care Visits
}

\author{
Hillary Samples, $P h D, M H S^{7}$, Elizabeth A. Stuart, $P h D^{2}$, Brendan Saloner, $P h D^{2}$, \\ Colleen L. Barry, PhD MPP', and Ramin Mojtabai, MD, $P h D, M P H^{2,3}$ \\ ${ }^{1}$ Columbia University Mailman School of Public Health, New York, NY, USA; ${ }^{2}$ Johns Hopkins Bloomberg School of Public Health, Baltimore, MD, USA; \\ 3Johns Hopkins University School of Medicine, Baltimore, MD, USA.
}

\begin{abstract}
BACKGROUND: Primary care providers encounter a large proportion of the population with depression. Yet, many primary care patients with depression remain undiagnosed and untreated.
\end{abstract}

OBJECTIVE: This study aims to examine depression screening patterns and the role of screening in depression diagnosis and treatment in the outpatient primary care setting.

DESIGN: This is a cross-sectional analysis of nationally representative survey data of visits to outpatient physician offices from the 2005 to 2015 National Ambulatory Medical Care Surveys.

PARTICIPANTS: The sample included the first visit in the past year to a primary care provider by patients 12 years and older $(N=16,887)$.

METHODS: The associations of visit characteristics with depression screening and of depression screening with depression diagnosis and treatment during the visit were assessed using logistic regression. Logistic regression with propensity score weighting was used to estimate the odds of depression diagnosis and treatment under the counterfactual scenario in which patients who visited providers with lower depression screening rates had visited providers with higher screening rates instead. All models were adjusted for patient and visit characteristics. KEY RESULTS: A small proportion of sample visits involved depression screening (3.0\%). Visits by patients with depressive symptom complaints were associated with higher odds of depression screening than other visits. When visits were weighted to have similar demographic and clinical characteristics, visits to providers with higher screening rates had higher odds of diagnosis $(\mathrm{OR}=1.99$, $p<0.001)$ and treatment $(\mathrm{OR}=1.61, p=0.001)$ compared to visits to providers with lower screening rates.

CONCLUSIONS: Physicians appear to use depression screening selectively based on patients' presenting symptoms. Higher screening rates were associated with higher odds of depression diagnosis and treatment, and even modest increases in screening rates could meaningfully

Electronic supplementary material The online version of this article (https://doi.org/10.1007/s11606-019-05192-3) contains supplementary material, which is available to authorized users.

Received May 18, 2018

Revised December 28, 2018

Accepted May 20, 2019

Published online August 6, 2019 increase population-level rates of depression identification and treatment in primary care. Future research is needed to identify barriers to depression care and implement systematic interventions to improve services and patient outcomes.

KEY WORDS: depression; screening; primary care.

J Gen Intern Med 35(1):12-20

DOI: $10.1007 / \mathrm{s} 11606-019-05192-3$

(C) Society of General Internal Medicine 2019

\section{BACKGROUND}

Primary care is the main access point to healthcare in the USA. ${ }^{1}$ Consequently, primary care providers encounter a large proportion of the population with depression and have assumed much of the responsibility for treating depression, mainly due to medication advances and the rise of managed care. ${ }^{2,3}$ Yet, estimates suggest that $75-85 \%$ of primary care patients with depression remain untreated. 4

Low depression treatment rates can partially be explained by low identification rates. A meta-analysis examining unaided depression diagnoses by general practitioners found that less than half of primary care patients with depression are correctly identified. ${ }^{4}$ However, patients who do not meet diagnostic criteria for depression are sometimes misdiagnosed with depression $^{4,6}$ and are increasingly treated, which could reflect misidentification of subclinical symptoms or impediments to proper diagnosis. ${ }^{7}$ Some evidence has shown that primary care doctors record diagnoses for patients' somatic complaints (e.g., fatigue, insomnia, or headache) instead of depression primarily because they are unsure about the diagnosis. 8,9

Diagnostic uncertainty reflects two central issues in mental healthcare. First, diagnosing mental illness is complicated. Depression diagnosis requires the presence of at least one of two key symptoms and a minimum of four additional symptoms out of nine total criteria, and symptoms must persist for at least 2 weeks. ${ }^{10,11}$ Adding to the complexity of diagnosing depression at a clinical threshold, the criteria include symptoms that healthy people experience to varying degrees in daily life. Second, many primary care physicians have limited training in mental 
healthcare, which may result in a lack of expertise and confidence. $^{12}$

Depression screeners were designed to reduce diagnostic uncertainty, and the United States Preventive Services Task Force (USPSTF) recommends routine screening for individuals $\geq$ 12 years based on evidence of the utility of screening in primary care settings. ${ }^{13}, 14$ The USPSTF acknowledges gaps in the evidence $^{15}$ and critics' concerns about inadequate or unnecessary treatment ${ }^{16}$ but concludes the benefits of screening outweigh potential harms. ${ }^{15}$ Nonetheless, depression screening is rare in primary care settings, suggesting that physicians screen patients only under certain circumstances or continue to rely on clinical judgment in their diagnoses. ${ }^{17,} 18$

The divergence between screening recommendations and practice motivated this study. Specifically, we aimed to examine (1) evidence for selective rather than universal screening, (2) the relationship of screening with depression diagnosis and treatment during the visit, and (3) the potential impact of increasing screening rates at the population level. We hypothesized that the odds of depression screening would be higher when patients present with depressive symptom complaints and that the odds of depression diagnosis and treatment would be higher when screening is conducted. We further hypothesized that visits to providers with higher screening rates would increase the odds of depression diagnosis and treatment.

\section{METHODS}

\section{Data}

Data were from the 2005 to 2015 National Ambulatory Medical Care Survey (NAMCS), a cross-sectional survey of outpatient visits to physician offices conducted annually by the National Center for Health Statistics (NCHS). The survey employs a multistage probability design, and sampling weights can be used to obtain nationally representative estimates of visits to officebased physicians.

\section{Sample}

The sample included visits with individuals $\geq 12$ years based on USPSTF recommendations. ${ }^{13,}{ }^{14}$ Visits were limited to providers specializing in general or family medicine, internal medicine, pediatrics, or obstetrics and gynecology. To assess screening as a detection rather than a monitoring tool, visits were included if the major reason for the visit was for a new problem or preventive care, while visits for chronic problems and surgery-related care were excluded. The sample was limited to visits by patients with no visits in the previous year to exclude visits by patients who could have been screened during a prior visit. Including earlier visits may artificially lower screening rates if, for example, screening is conducted on an annual basis and not during follow-up visits.

\section{Measures}

NAMCS has unique data on patients' reasons for seeking care, which are coded using a classification system specifically designed for NCHS. ${ }^{19}$ The categories were tested on the basis of their performance identifying patients' reasons for seeking care as well as providers' responses to patient complaints. ${ }^{19}$ NAMCS recorded patient complaints, symptoms, or other reasons for the visit in the patient's own words, and we categorized the primary complaint as a depressive symptom if it was consistent with depression criteria in the Diagnostic and Statistical Manual of Mental Disorders, 4th edition (DSM-IV). ${ }^{10}$ Symptom categories included depression (e.g., sadness, hopelessness), tiredness/ exhaustion (e.g., fatigue, lack of energy), abnormal appetite (e.g., excessive, decreased), weight gain or loss, anxiety/ nervousness (e.g., being upset, worried), sleep disturbances (e.g., trouble falling asleep, sleepiness), restlessness, memory problems (e.g., forgetfulness), lack of libido (e.g., loss of sex drive), self-esteem problems (e.g., guilt), and other mental healthrelated symptoms (e.g., cannot cope, danger to self).

Depression screening was recorded using a checklist of diagnostic and screening services ordered or provided during the visit. Due to the checklist format, a documented screening did not include detail on the specific screening method or tool used. Provider screening rates were calculated as the number of visits with a depression screening out of all visits sampled for each provider.

One to three diagnoses were recorded for each patient visit based on the International Classification of Diseases, Ninth Revision, Clinical Modification (ICD-9$\mathrm{CM}){ }^{20}$ An additional item asked, "Regardless of the diagnoses previously listed, does the patient now have depression?" Depression diagnosis was defined as either an ICD-9-CM code for depression corresponding to DSM-IV depression diagnoses $(296.2,296.3,300.4$, and 311) or a response to the additional item indicating the patient has depression.

Medication and nonmedication treatment were recorded if they were ordered or provided during the visit. Depression treatment was defined as either therapy (psychotherapy, other mental health counseling) or antidepressant medications. All medications were coded by NCHS according to their therapeutic classification using a proprietary database. ${ }^{21}$ Antidepressants included selective serotonin reuptake inhibitors, tricyclic antidepressants, monoamine oxidase inhibitors, tetracyclic antidepressants, selective serotonin-norepinephrine reuptake inhibitors, phenylpiperazine antidepressants, and miscellaneous antidepressants.

Patient demographic and clinical characteristics included sex, age, race/ethnicity, insurance, census region, urban/rural area (measured by metropolitan statistical area), and the presence of any comorbid chronic condition (arthritis, asthma, cancer, cerebrovascular disease, congestive heart failure, chronic renal failure, chronic 
obstructive pulmonary disease, diabetes, hyperlipidemia, hypertension, ischemic heart disease, obesity, or osteoporosis). Encounter characteristics included patient status (new or established), physician status as the patient's primary care provider, visit type (preventive care or new problem), and the year in which the visit occurred.

\section{Analyses}

Data were analyzed using the svy suite of commands in Stata statistical software, Version $15 .^{22}$ NCHS guidelines were followed to account for the complex, multistage sampling design, incorporating patient visit weights to obtain estimates that are nationally representative of outpatient physician office visits. $^{23}$

To assess evidence for selective screening practices, we used logistic regression to estimate the relationship between depression screening and patient characteristics, comparing patients reporting a depressive symptom as their primary complaint to those reporting other reasons for the visit. To examine the relationship of screening with diagnosis and treatment, we used separate logistic regression models for each outcome, comparing visits with and without screening. Regression-adjusted predicted probabilities were computed using Stata's margins command to compare screening for those reporting depressive symptoms to those reporting other reasons for the visit and to assess whether the relationship between screening and depression diagnosis and treatment differed based on patients' primary complaints. All models were adjusted for the patient and encounter characteristics described above.

To examine the population-level impact of screening rates on depression diagnosis and treatment, we estimated the change in the number of diagnoses and treatments if patients who visited office-based physicians with lower screening rates had instead visited providers with higher screening rates. Screening rates were calculated among the visits sampled for each provider and summarized using survey weights to obtain estimates that are representative of outpatient visits at the physician level. The distribution of provider screening rates was used to define providers with higher $(\geq 95$ th percentile) and lower $(<95$ th percentile) screening rates and then categorize patient visits to these groups. To account for potential differences in the patient populations visiting providers screening at higher vs. lower rates, propensity score weighting was used. Propensity scores were calculated from a logistic regression model predicting the probability of visiting a provider with a lower screening rate based on characteristics that may influence screening, including direct measures of health (any chronic condition and depressive symptom complaints) and characteristics that may be related to health status and healthcare utilization (sex, age, race/ethnicity, insurance, census region, and urban/rural area). Propensity score estimates incorporated survey sampling weights but no other survey design variables (i.e., strata and clusters). ${ }^{24}$
Weighting by the odds was implemented using propensity scores to calculate weights and then multiplying weights by the survey sampling weights to obtain new weights to make visits to providers with higher screening rates comparable to visits to providers with lower screening rates. ${ }^{24}$ This weighting scheme allowed us to examine the counterfactual scenario in which patients who visited providers with lower screening rates had instead visited providers with higher screening rates.

\section{RESULTS}

Survey-weighted visit characteristics are presented in Table 1. The sample $(N=16,887)$ comprised visits by patients who were mostly female $(64.7 \%)$, white $(69.4 \%)$, privately insured $(64.8 \%)$, and living in more urban areas (89.4\%). Depression screening occurred in $3.0 \%$ of visits overall. Depressive symptoms were reported in $7.8 \%$ of visits in which screening occurred and $2.1 \%$ of visits without screening. Depression was identified and treated in 6.2 and $7.2 \%$ of visits, respectively.

\section{Correlates of Depression Screening}

Results for depression screening are presented in the left panel of Table 2. Visits were associated with higher odds of depression screening if a depressive symptom was the primary complaint $(\mathrm{OR}=5.32, p<0.001)$. Other variables associated with screening included comorbid chronic conditions, sex, the physician being the primary care provider for the patient, and visit type (Table 2).

\section{Association of Screening with Depression Diagnosis and Treatment}

Results for depression diagnosis and treatment are presented in the middle and right panels of Table 2, respectively. Visits were associated with higher odds of depression diagnosis if screening was conducted $(\mathrm{OR}=4.20, p<0.001)$ or if a depressive symptom was the primary complaint $(\mathrm{OR}=7.63$, $p<0.001)$. The association between screening and the probability of depression diagnosis was stronger for visits with depressive symptom complaints than for visits with other complaints (Fig. 1). Other variables associated with depression diagnosis included comorbid chronic conditions, sex, age, race, insurance, and region (Table 2).

Visits were associated with higher odds of depression treatment if screening was conducted $(\mathrm{OR}=1.82, p=0.01)$ or if a depressive symptom was the primary complaint $(\mathrm{OR}=3.31$, $p<0.001)$. The association between screening and the probability of depression treatment did not differ for visits with and without depressive symptom complaints. Other variables associated with depression treatment included depression diagnosis, sex, age, race, insurance, region, and visit type (Table 2). 
Table 1 Descriptive Characteristics of All Sample Patient Visits to Primary Care Physician Offices, Stratified by Screening Status

\begin{tabular}{|c|c|c|c|c|}
\hline & \multirow{2}{*}{$\frac{\text { Total }}{N(\%)}$} & \multirow{2}{*}{$\frac{\text { Screened }}{N(\%)}$} & \multirow{2}{*}{$\frac{\text { Unscreened }}{N(\%)}$} & \multirow[t]{3}{*}{$p$} \\
\hline & & & & \\
\hline & 16,887 & $491(2.9)$ & $16,396(97.1)$ & \\
\hline \multicolumn{5}{|l|}{ Depression care } \\
\hline Depression diagnosis & $1122(6.2)$ & $127(21.9)$ & $995(5.7)$ & $<0.001$ \\
\hline Depression treatment & $1324(7.2)$ & $111(19.9)$ & $1213(6.8)$ & $<0.001$ \\
\hline \multicolumn{5}{|l|}{ Clinical characteristics } \\
\hline Depressive symptoms & $388(2.3)$ & $46(7.8)$ & $342(2.1)$ & 0.001 \\
\hline Chronic comorbidities & $5897(34.5)$ & $206(42.2)$ & $5691(34.3)$ & 0.02 \\
\hline \multicolumn{5}{|l|}{ Demographic characteristics } \\
\hline \multicolumn{5}{|c|}{ Sex } \\
\hline Female & $11,177(64.7)$ & $369(71.5)$ & $10,808(64.5)$ & 0.04 \\
\hline \multicolumn{5}{|l|}{ Age } \\
\hline $12-17$ & $1822(9.9)$ & $43(9.8)$ & $1779(9.9)$ & 0.97 \\
\hline $18-24$ & $2053(13.0)$ & $46(9.5)$ & 2007 (13.1) & 0.03 \\
\hline $25-34$ & $3005(18.3)$ & 78 (17.4) & $2927(18.3)$ & 0.68 \\
\hline $35-44$ & $2983(18.4)$ & $94(20.9)$ & $2889(18.3)$ & 0.36 \\
\hline $45-54$ & $2780(16.6)$ & $88(16.3)$ & $2692(16.6)$ & 0.91 \\
\hline $55-64$ & $2269(13.2)$ & $81(15.4)$ & $2188(13.1)$ & 0.30 \\
\hline $65+$ & 1975 (10.6) & $61(10.7)$ & 1914 (10.6) & 0.96 \\
\hline \multicolumn{5}{|l|}{ Race/ethnicity } \\
\hline White & $12,212(69.4)$ & $354(68.7)$ & $11,858(69.4)$ & 0.85 \\
\hline Black & $1740(11.4)$ & $46(10.6)$ & $1694(11.4)$ & 0.63 \\
\hline Hispanic & 2017 (13.3) & 64 (13.6) & $1953(13.3)$ & 0.89 \\
\hline Other & $918(5.9)$ & $27(7.1)$ & $891(5.8)$ & 0.68 \\
\hline \multicolumn{5}{|l|}{ Insurance } \\
\hline Private & $10,645(64.8)$ & $313(65.1)$ & $10,332(64.8)$ & 0.96 \\
\hline Medicaid & $1400(8.0)$ & $44(7.8)$ & $1356(8.1)$ & 0.87 \\
\hline Medicare & $1779(9.7)$ & $55(10.5)$ & $1724(9.7)$ & 0.69 \\
\hline Self pay & $1150(7.6)$ & $38(7.2)$ & $1112(7.6)$ & 0.83 \\
\hline Other & $1913(9.9)$ & $41(9.5)$ & $1872(9.9)$ & 0.90 \\
\hline \multicolumn{5}{|l|}{ Census region } \\
\hline Northeast & $2289(16.9)$ & $86(25.9)$ & $2203(16.6)$ & 0.07 \\
\hline Midwest & $4286(20.2)$ & $123(19.7)$ & $4163(20.2)$ & 0.88 \\
\hline South & $6050(39.2)$ & $175(38.7)$ & $5875(39.2)$ & 0.94 \\
\hline West & $4262(23.8)$ & $107(15.6)$ & $4155(24.0)$ & 0.004 \\
\hline \multicolumn{5}{|l|}{ MSA } \\
\hline Urban & $14,688(89.4)$ & $440(92.2)$ & $14,248(89.3)$ & 0.18 \\
\hline \multicolumn{5}{|l|}{ Encounter characteristics } \\
\hline Patient status & & & & \\
\hline New patient & $9878(60.2)$ & $260(54.4)$ & $9618(60.4)$ & 0.12 \\
\hline \multicolumn{5}{|l|}{ Provider status } \\
\hline Patient's PCP & $7046(43.1)$ & $228(51.8)$ & $6818(42.8)$ & 0.04 \\
\hline \multicolumn{5}{|l|}{ Visit type } \\
\hline Preventive care & $7211(41.5)$ & $293(63.8)$ & $6918(40.9)$ & $<0.001$ \\
\hline
\end{tabular}

Percentages are survey-weighted and may not add to $100 \%$

MSA metropolitan statistical area, PCP primary care provider

\section{Projected Prevalence of Depression Diagnosis and Treatment Based on Provider Screening Rates}

To examine the population-level impact of screening rates on depression diagnosis and treatment, we estimated the change in the number of diagnoses and treatments if patients who visited providers with lower screening rates had instead visited providers with higher screening rates. Providers varied considerably in the frequency of depression screening within their sampled visits. On average, providers $(N=4476)$ screened in $3.2 \%$ of their sampled visits, but providers with higher screening rates $(\geq 95$ th percentile) screened in $\geq 5.0 \%$ of their visits $(N=286)$.

Weighting by the odds balanced the characteristic of visits to providers with lower and higher screening rates (Table S1 and Fig. S1, Online Supplement). Before weighting, a higher proportion of visits to providers with higher screening rates were with patients who reported a depressive symptom as the primary reason for the visit ( $p=0.01$; Table $\mathrm{S} 1)$. After propensity score weighting, there were no significant differences between visits to providers with higher and lower screening rates.

Results for depression diagnosis and treatment comparing propensity score-weighted visits to providers who screened at higher vs. lower rates are presented in Table 3. Visiting providers who screened at higher rates was associated with increased odds of depression diagnosis $(\mathrm{OR}=1.99, p<0.001)$ and treatment $(\mathrm{OR}=1.61, p=0.001)$, corresponding to a 4.8 percentage-point higher probability of depression diagnosis (95\% CI $=2.9-6.8, p<0.001)$ and a 5.3 percentage-point higher probability of depression treatment $(95 \% \mathrm{CI}=3.1-$ $7.5, p<0.001)$ compared to visits to providers with lower screening rates (Fig. 2). At the national level among the 46.5 million visits to primary care providers with lower 
Table 2 Adjusted Logistic Regression Results for Depression Screening, Diagnosis, and Treatment Among All 16,887 Sample Patient Visits to Primary Care Physician Offices

\begin{tabular}{|c|c|c|c|c|c|c|}
\hline & \multicolumn{2}{|c|}{ Depression screening } & \multicolumn{2}{|c|}{ Depression diagnosis } & \multicolumn{2}{|l|}{ Depression treatment } \\
\hline & OR $(95 \% \mathrm{CI})$ & $p$ & OR $(95 \% \mathrm{CI})$ & $p$ & OR $(95 \% \mathrm{CI})$ & $p$ \\
\hline \multicolumn{7}{|l|}{ Depression care } \\
\hline Depression screening & & & $4.20(3.05-5.80)$ & $<0.001$ & $1.82(1.19-2.78)$ & 0.01 \\
\hline Depression diagnosis & & & & & $14.77(11.96-18.25)$ & $<0.001$ \\
\hline \multicolumn{7}{|l|}{ Clinical characteristics } \\
\hline Depressive symptoms & $5.32(3.27-8.64)$ & $<0.001$ & $7.63(5.46-10.67)$ & $<0.001$ & $3.31(2.12-5.16)$ & $<0.001$ \\
\hline Chronic comorbidities & $1.42(1.08-1.86)$ & 0.01 & $2.04(1.70-2.45)$ & $<0.001$ & $1.16(0.97-1.39)$ & 0.10 \\
\hline \multicolumn{7}{|l|}{ Demographics } \\
\hline \multicolumn{7}{|l|}{ Sex } \\
\hline Male & 1.00 & - & 1.00 & - & 1.00 & - \\
\hline Female & $1.37(1.02-1.83)$ & 0.03 & $1.88(1.51-2.34)$ & $<0.001$ & $1.48(1.24-1.78)$ & $<0.001$ \\
\hline \multicolumn{7}{|l|}{ Age } \\
\hline $12-17$ & $1.40(0.80-2.46)$ & 0.24 & $0.43(0.26-0.71)$ & 0.001 & $0.42(0.27-0.66)$ & $<0.001$ \\
\hline $18-24$ & 1.00 & - & 1.00 & - & 1.00 & - \\
\hline $25-34$ & $1.28(0.84-1.97)$ & 0.25 & $1.45(1.01-2.08)$ & 0.05 & $1.28(0.94-1.74)$ & 0.12 \\
\hline $35-44$ & $1.44(0.92-2.26)$ & 0.11 & $1.67(1.23-2.28)$ & 0.001 & $1.57(1.17-2.10)$ & 0.003 \\
\hline $45-54$ & $1.21(0.75-1.95)$ & 0.44 & $1.54(1.11-2.13)$ & 0.01 & $2.01(1.47-2.75)$ & $<0.001$ \\
\hline $55-64$ & $1.28(0.79-2.06)$ & 0.31 & $1.41(0.96-2.09)$ & 0.08 & $1.45(1.04-2.01)$ & 0.03 \\
\hline $65+$ & $1.08(0.62-1.89)$ & 0.79 & $0.82(0.51-1.32)$ & 0.41 & $1.24(0.79-1.94)$ & 0.35 \\
\hline \multicolumn{7}{|l|}{ Race/ethnicity } \\
\hline White & 1.00 & - & 1.00 & - & 1.00 & - \\
\hline Black & $0.87(0.59-1.27)$ & 0.46 & $0.56(0.41-0.78)$ & 0.001 & $0.64(0.47-0.87)$ & 0.004 \\
\hline Hispanic & $1.08(0.73-1.62)$ & 0.69 & $0.74(0.55-1.01)$ & 0.06 & $0.95(0.70-1.30)$ & 0.77 \\
\hline Other & $1.36(0.57-3.25)$ & 0.49 & $0.45(0.28-0.73)$ & 0.001 & $0.58(0.39-0.85)$ & 0.01 \\
\hline \multicolumn{7}{|l|}{ Insurance } \\
\hline Private & 1.00 & - & 1.00 & - & 1.00 & - \\
\hline Medicaid & $0.96(0.58-1.60)$ & 0.89 & $1.75(1.27-2.42)$ & 0.001 & $0.88(0.62-1.24)$ & 0.46 \\
\hline Medicare & $1.16(0.74-1.81)$ & 0.51 & $2.16(1.48-3.15)$ & $<0.001$ & $1.13(0.78-1.62)$ & 0.53 \\
\hline Self pay & $1.06(0.61-1.86)$ & 0.83 & $0.89(0.56-1.43)$ & 0.64 & $0.47(0.29-0.77)$ & 0.003 \\
\hline Other & $1.17(0.57-2.39)$ & 0.67 & $1.14(0.86-1.50)$ & 0.36 & $0.60(0.43-0.82)$ & 0.001 \\
\hline \multicolumn{7}{|l|}{ Census region } \\
\hline Northeast & 1.00 & - & 1.00 & - & 1.00 & - \\
\hline Midwest & $0.69(0.39-1.24)$ & 0.22 & $1.66(1.12-2.45)$ & 0.01 & $1.72(1.31-2.25)$ & $<0.001$ \\
\hline South & $0.72(0.40-1.31)$ & 0.28 & $1.23(0.85-1.78)$ & 0.27 & $1.42(1.09-1.83)$ & 0.01 \\
\hline West & $0.44(0.23-0.81)$ & 0.01 & $1.47(1.02-2.12)$ & 0.04 & $1.48(1.11-1.98)$ & 0.01 \\
\hline \multicolumn{7}{|l|}{ MSA } \\
\hline Rural & 1.00 & - & 1.00 & - & 1.00 & - \\
\hline Urban & $1.50(0.88-2.56)$ & 0.14 & $0.97(0.74-1.28)$ & 0.84 & $0.94(0.70-1.26)$ & 0.67 \\
\hline \multicolumn{7}{|l|}{ Encounter characteristics } \\
\hline \multicolumn{7}{|l|}{ Patient status } \\
\hline Established patient & 1.00 & - & 1.00 & - & 1.00 & - \\
\hline New patient & $1.01(0.71-1.43)$ & 0.96 & $1.10(0.92-1.32)$ & 0.28 & $0.99(0.82-1.20)$ & 0.92 \\
\hline \multicolumn{7}{|l|}{ Provider status } \\
\hline Not the patient's PCP & 1.00 & - & 1.00 & - & 1.00 & - \\
\hline Patient's PCP & $1.45(1.03-2.04)$ & 0.03 & $1.17(0.96-1.43)$ & 0.12 & $1.18(0.95-1.45)$ & 0.13 \\
\hline \multicolumn{7}{|l|}{ Visit type } \\
\hline New problem & 1.00 & - & 1.00 & - & 1.00 & - \\
\hline Preventive care & $2.66(1.96-3.60)$ & $<0.001$ & $0.95(0.79-1.15)$ & 0.60 & $1.21(1.03-1.42)$ & 0.02 \\
\hline Year & $0.96(0.89-1.04)$ & 0.32 & $1.04(1.00-1.08)$ & 0.04 & $1.02(0.99-1.05)$ & 0.26 \\
\hline
\end{tabular}

All estimates are adjusted for patient sex, age, racelethnicity, insurance, census region, urban/rural status, any comorbid chronic condition, patient status, physician status as the patient's primary care provider, visit type, and year. Estimates for diagnosis are further adjusted for depression screening. Estimates for treatment are further adjusted for depression screening and diagnosis

MSA metropolitan statistical area, PCP primary care provider

screening rates, a 4.8 percentage-point increase in depression diagnosis and a 5.3 percentage-point increase in depression treatment would translate into an additional 2.2 million visits each year with a diagnosis and 2.4 million visits each year with treatment.

\section{DISCUSSION}

\section{Main Findings}

There were three main findings in this study. First, depression screening rates were low. Only $3 \%$ of sampled visits to outpatient primary care physician offices for new problems or preventive care included screening. This is consistent with prior studies analyzing NAMCS data, which showed that screening was documented in $1.8-3.4 \%$ of visits to primary care providers ${ }^{25,26}$ and that overall screening rates for adult visits to nonpsychiatrists have increased over time to $3 \%$ in $2015 .{ }^{27}$ These low screening rates suggest that providers may rely on their clinical judgment to identify depression. ${ }^{28,} 29$ Others might overlook depression altogether due to resource or time constraints, as evidence has shown that screening occurs more often than not in longer visits. ${ }^{26}$ However, twoquestion screeners have been validated in both adult and 


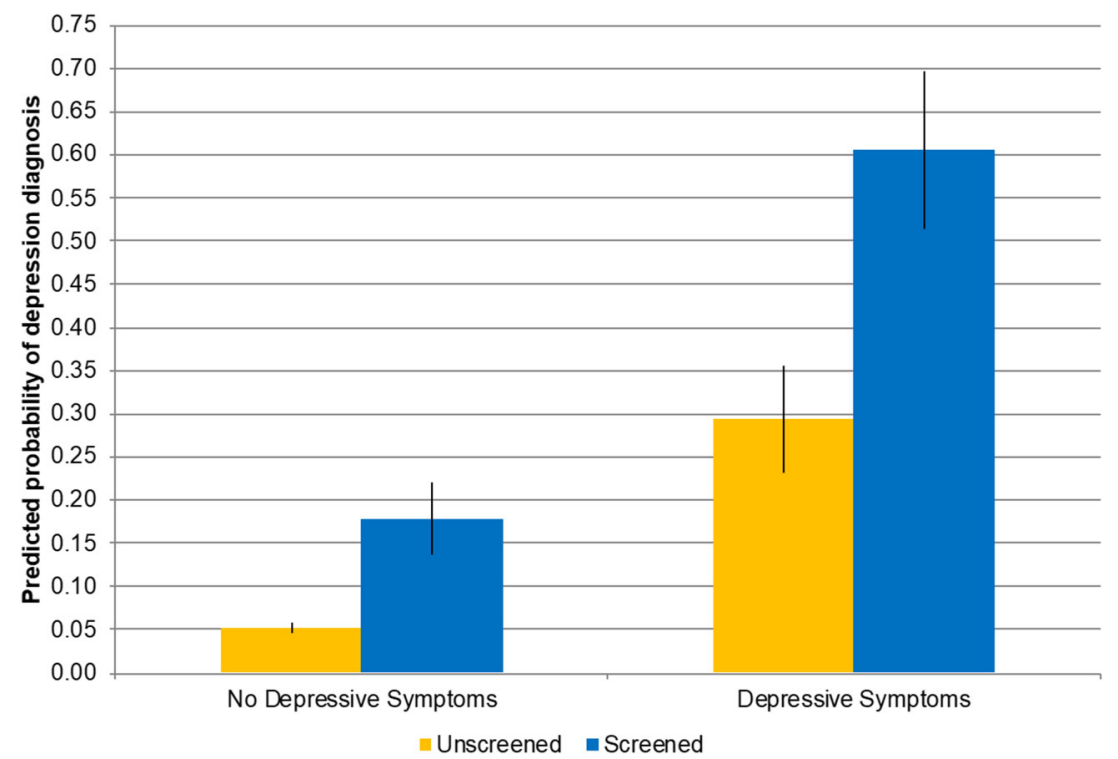

Figure 1 Regression-adjusted predicted probability of depression diagnosis among visits with and without depressive symptoms, stratified by screening status. Note: All estimates are adjusted for patient sex, age, race/ethnicity, insurance, census region, urban/rural status, any comorbid chronic condition, patient status, physician status as the patient's primary care provider, visit type, and year.

adolescent populations to accommodate primary care providers' many responsibilities and brief visit times. ${ }^{30,31}$

Second, the association between depressive symptoms and screening indicates that providers tend to conduct selective screening with patients who are more likely to meet the criteria for depression. Screening was associated with higher odds of depression diagnosis and treatment independent of patients' primary complaints, but the relationship between depression screening and diagnosis was stronger when patients reported depressive symptoms. This could indicate that in the absence of screening, depressive symptoms are less likely to be recognized as indicators of possible depression. Alternatively, patients reporting depressive symptoms may simply be more likely to have depression, in which case screening tools perform better. ${ }^{32}$

Third, screening was associated with higher odds of depression diagnosis and treatment. Furthermore, propensity-weighted analyses indicated that the probability of depression diagnosis and treatment would be higher if patients who visited providers with lower screening rates had visited providers with higher screening rates instead, suggesting that the prevalence of depression identification and treatment could be substantially higher if providers had higher screening rates regardless of their case-mix or patients' presenting complaints.

Table 3 Adjusted Logistic Regression Results for Depression Diagnosis and Treatment, Comparing Propensity Score-Weighted Visits to Providers Who Screen at Higher Rates with Visits to Providers Who Screen at Lower Rates

\begin{tabular}{|c|c|c|c|c|}
\hline & \multicolumn{2}{|c|}{ Depression diagnosis } & \multicolumn{2}{|c|}{ Depression treatment } \\
\hline & OR $(95 \% \mathrm{CI})$ & $p$ value & OR $(95 \% \mathrm{CI})$ & $p$ value \\
\hline \multicolumn{5}{|l|}{ Depression care } \\
\hline \multicolumn{5}{|l|}{ Depression screening } \\
\hline Lower-rate provider & 1.00 & - & 1.00 & - \\
\hline Higher-rate provider & $1.99(1.57-2.54)$ & $<0.001$ & $1.61(1.23-2.11)$ & 0.001 \\
\hline Depression diagnosis & & & $11.65(8.25-16.44)$ & $<0.001$ \\
\hline \multicolumn{5}{|l|}{ Encounter characteristics } \\
\hline \multicolumn{5}{|l|}{ Patient status } \\
\hline Established patient & 1.00 & - & 1.00 & - \\
\hline New patient & $1.05(0.77-1.43)$ & 0.75 & $1.30(0.98-1.72)$ & 0.07 \\
\hline \multicolumn{5}{|l|}{ Provider status } \\
\hline Not the patient's PCP & 1.00 & _- & 1.00 & - \\
\hline Patient's PCP & $1.20(0.90-1.59)$ & 0.22 & $1.32(0.96-1.81)$ & 0.08 \\
\hline \multicolumn{5}{|l|}{ Visit type } \\
\hline New problem & 1.00 & - & 1.00 & - \\
\hline Preventive care & $0.84(0.62-1.14)$ & 0.27 & $1.16(0.90-1.49)$ & 0.25 \\
\hline Year & $1.04(0.99-1.09)$ & 0.11 & $1.03(0.97-1.08)$ & 0.32 \\
\hline
\end{tabular}

Propensity scores are adjusted for patient sex, age, racelethnicity, insurance, census region, urban/rural status, and any comorbid chronic condition. All estimates are adjusted for patient sex, age, race/ethnicity, insurance, census region, urban/rural status, any comorbid chronic condition, patient status, physician status as the patient's primary care provider, visit type, and year. Estimates for treatment are further adjusted for depression diagnosis MSA metropolitan statistical area, PCP primary care provider 


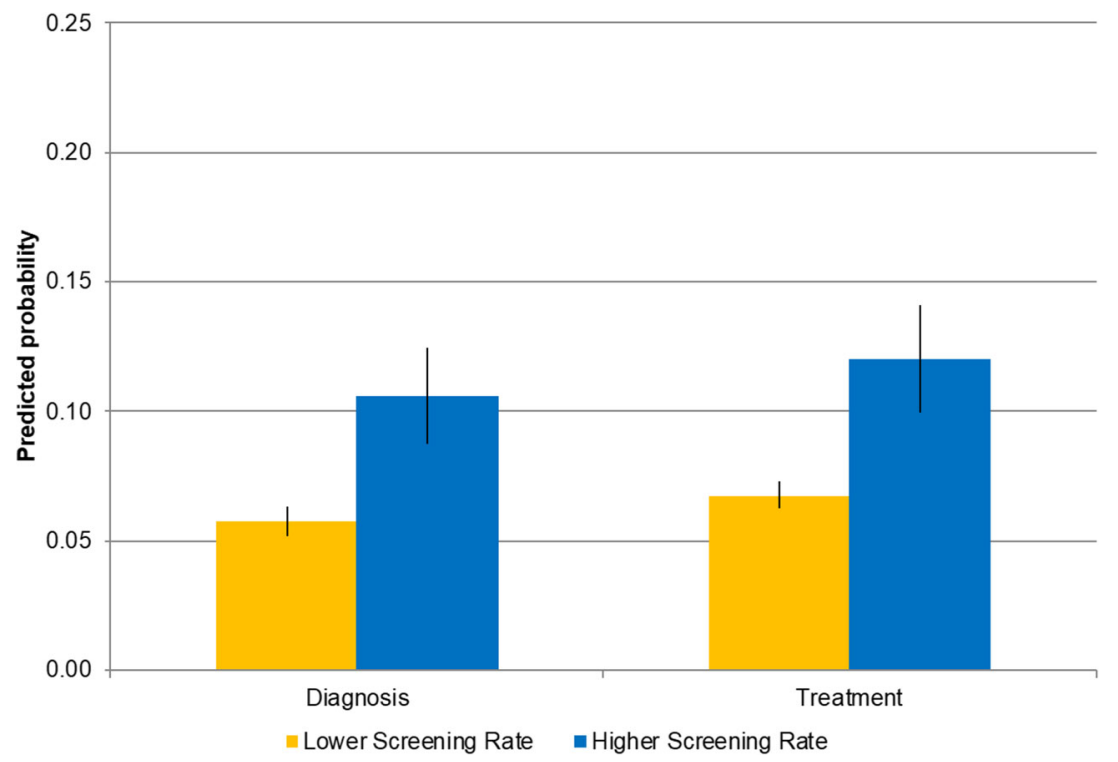

Figure 2 Regression-adjusted predicted probability of depression diagnosis and treatment, stratified by provider screening rates. Note: All estimates are adjusted for patient sex, age, race/ethnicity, insurance, census region, urban/rural status, any comorbid chronic condition, patient status, physician status as the patient's primary care provider, visit type, and year. Estimates for treatment are further adjusted for depression diagnosis.

\section{Limitations and Strengths}

These data uniquely allow for analyses at both the physician and patient visit levels to produce results that are nationally representative of outpatient visits to primary care providers. However, very limited information on provider or practice characteristics were available. Provider demographics, training, experience, and practice organization that may explain variations in practice style were not available, and practice characteristics may be particularly important considering that the USPSTF recommendations rely on the presence of systems and staff that can ensure proper screening, diagnosis, and treatment or referral for evidence-based care. $^{13}$

While important patient characteristics could be included in the analyses, other characteristics that could influence providers' patient populations and their decisions to use depression screening were not measured, potentially affecting the extent to which groups were truly comparable. For example, lifestyle factors (e.g., exercise and tobacco use) or the patient's affect may influence the provider's decision to use depression screening. NAMCS does include measures for tobacco use and body mass index, but these could not be included due to a high proportion of missing values. Additionally, information about patients' socioeconomic status (e.g., education, income, or occupation) might have improved comparability between patient populations.

Another limitation was the inability to determine the specific screening method used or the results of screening, which could be used to evaluate the appropriateness of depression diagnosis and the need for treatment based on these data. Thus, the extent to which higher diagnosis rates arise from falsepositive screening and higher treatment rates represent unnecessary treatment could not be determined.
Although we excluded patients with prior visits in the past year and visits for ongoing health problems, the temporal of order of depressive symptoms, screening, diagnosis, and treatment cannot be established due to the cross-sectional nature of the data. For example, it is possible that patients with chronic depression were screened for monitoring of symptoms.

Finally, separate sensitivity analyses showed the same pattern of results as the main analyses when the sample was not restricted to (1) visits for new problems or preventive care (Appendix 1, Online Supplement) or (2) the first visit in the past year (Appendix 2, Online Supplement).

\section{CONCLUSION}

Significant progress has been made to improve depression care in primary care settings, but important challenges remain. ${ }^{33}$ Universal depression screening in primary care has been recommended as a way to improve depression detection and to reduce disparities in care. ${ }^{34}$ However, the evidence presented here suggests that universal screening may not be feasible in primary care settings and that selective depression screening based on presenting symptoms might instead be the typical practice. Though physicians generally find depression screening helpful, ${ }^{15}$ primary care settings often have limited personnel, time, and other resources to address common mental health problems. ${ }^{35}$ More research is needed to identify practice-level barriers to comprehensive screening.

While screening practices in this study were associated with significantly greater odds of diagnosis and treatment, the high prevalence of undetected and untreated depression in primary care underscores the need for strategies to 
improve depression care in these settings. The results of this study also highlight the potential impact of such strategies at the population level since even modest increases in screening rates were found to meaningfully increase rates of depression identification and treatment in primary care. Depression care often requires support services, which may not be available in many primary care settings. ${ }^{35,}{ }^{36}$ However, measurement-based decision support systems that can be implemented in primary care settings with many competing demands have been shown to improve the quality of depression care and patient outcomes to levels comparable with specialty psychiatric settings. ${ }^{37}$ Future studies may help to develop approaches that encourage adoption of such tools and more widespread system improvements.

Corresponding Author: Hillary Samples, PhD, MHS; Columbia University Mailman School of Public Health, New York, NY, USA (e-mail: h.samples@columbia.edu).

Funding Information Financial support for this work was provided by grants from the Agency for Healthcare Research and Quality (AHRQ) [grant number: T32 HSO00029] and the Institute of Education Sciences (IES) [grant number: R305D150001; PIs: Stuart and Dong] and awards from the Johns Hopkins Bloomberg School of Public Health Department of Health Policy and Management.

\section{Compliance with Ethical Standards:}

Conflict of Interest: The authors declare that they do not have a conflict of interest.

\section{REFERENCES}

1. Agency for Healthcare Research and Quality; Medical Expenditure Panel Survey (MEPS), Table 2.1: People Who Have a Usual Primary Care Provider, by Race/Ethnicity, United States, 2014. Rockville, MD; 2014. https://meps.ahrq.gov/data_stats/summ_tables/hc/acc/2014/ acctocare_2_1_2014.htm. Accessed 19 April 2018.

2. Wang PS, Demler O, Olfson M, Pincus HA, Wells KB, Kessler RC. Changing profiles of service sectors used for mental health care in the United States. Am J Psychiatry 2006; 163(7):1187-1198. doi:https://doi. org/10.1176/ajp.2006.163.7.1187.

3. Olfson M, Marcus SC, Druss B, Elinson L, Tanielian T, Pincus HA National trends in the outpatient treatment of depression. JAMA 2002;287(2):203. doi:https://doi.org/10.1001/jama.287.2.203.

4. Mitchell AJ, Vaze A, Rao S. Clinical diagnosis of depression in primary care: a meta-analysis. Lancet 2009;374:609-619. doi:https://doi.org/10. 1016/S0140.

5. Pence BW, O'Donnell JK, Gaynes BN. The depression treatment cascade in primary care: a public health perspective. Curr Psychiatry Rep 2012;14:328-335. doi:https://doi.org/10.1007/s11920-012-0274y.

6. Mojtabai $\mathbf{R}$ Clinician-identified depression in community settings: concordance with structured-interview diagnoses. Psychother Psychosom 2013;82:161-169. doi:https://doi.org/10.1159/000345968.

7. Mojtabai R, Olfson M. Proportion of antidepressants prescribed without a psychiatric diagnosis is growing. Health Aff 2011;30(8):1434-1442. doi:https://doi.org/10.1377/hlthaff.2010.1024.

8. Rushton JL, Felt BT, Roberts MW. Coding of pediatric behavioral and mental disorders. Pediatrics. 2002;110(1).

9. Rost K, Smith GR, Matthews DB, Guise B. The deliberate misdiagnosis of major depression in primary care. Arch Fam Med 1994;3:333-337.
10. American Psychiatric Association. Diagnostic and Statistical Manual of Mental Disorders: DSM-IV, 4th Ed., Text Revision. 4th edn. Arlington: American Psychiatric Association; 2000.

11. American Psychiatric Association. Diagnostic and Statistical Manual of Mental Disorders: DSM-5, 5th Ed. 5th ed. Arlington: American Psychiatric Association; 2013.

12. Leigh H, Stewart D, Mallios R. Mental health and psychiatry training in primary care residency programs B Part II. What skills and diagnoses are taught, how adequate, and what affects training directors' satisfaction? Gen Hosp Psychiatry 2006;28:195-204. doi:https://doi.org/10.1016/j. genhosppsych.2005.10.004.

13. Siu AL, Bibbins-Domingo K, Grossman DC, et al. Screening for depression in adults: US Preventive Services Task Force recommendation statement. JAMA 2016;315(4):380-387. doi:https://doi.org/10.1001/ jama.2015.18392.

14. Siu AL, U.S. Preventive Services Task Force. Screening for depression in children and adolescents: US Preventive Services Task Force recommendation statement. Pediatrics 2016;137(3):1-8. doi:https://doi.org/10. 1542/peds.2015-4467.

15. O'Connor E, Rossom RC, Henninger M, et al. Screening for Depression in Adults: An Updated Systematic Evidence Review for The U.S. Preventive Services Task Force. Rockville: Agency for Healthcare Research and Quality; 2015.

16. Thombs BD, Ziegelstein RC, Roseman M, Kloda LA, Ioannidis JP. There are no randomized controlled trials to support the United States Preventive Services Task Force guideline on screening for depressionin primary care: a systematic review. BMC Med. 2014;12:13. doi:https:// doi.org/10.1186/1741-7015-12-13.

17. U.S. Department of Health \& Human Services: Office of the Assistant Secretary for Planning and Evaluation. Percentage of Primary Care Physician Office Visits that Include Depression Screening. Health System Measurement Project. https://healthmeasures.aspe.hhs.gov/measure/ 258. Accessed 12 March 2018.

18. U.S. Preventive Services Task Force. Screening for depression in adults: U.S. Preventive Services Task Force recommentaion statement. Ann Intern Med. 2009;151(11):785-792.

19. Schneider D, Appleton L, McLemore T. A Reason for Visit Classification for Ambulatory Care. Vol 2. Hyattsville: National Center for Health Statistics; 1979. https://www.cdc.gov/nchs/data/series/sr_02/sr02 078.pdf. Accessed 12 Oct 2018.

20. World Health Organization. International Classification of Diseases, 9th Revision, Clinical Modification (ICD-9-CM). Geneva: World Health Organization; 2008.

21. Multum. Denver, CO: Cerner Corporation https://www.cerner.com/ solutions/drug-database. Accessed 19 April 2018.

22. StataCorp. Stata Statistical Software: Release 15. 2017.

23. Using Ultimate Cluster Models with NAMCS and NHAMCS Public Use Files. National Center for Health Statistics, Centers for Disease Control and Prevention, U.S. Department of Health and Human Services; 2004. https://www.cdc.gov/nchs/data/ahcd/ultimatecluster.pdf. Accessed 19 April 2018.

24. Dugoff EH, Schuler M, Stuart EA. Generalizing observational study results: applying propensity score methods to complex surveys. Health Serv Res 2014;49(1):284-303. doi:https://doi.org/10.1111/1475-6773. 12090.

25. McGoey ST, Huang KE, Palmes GK. Low depression screening rates in U.S. ambulatory care. Psychiatr Serv. 2013;64(10):1068

26. Schmitt MR, Miller MJ, Harrison DL, Touchet BK. Relationship of depression screening and physician office visit duration in a national sample. Psychiatr Serv 2010;61(11):1126-1131.

27. Bhattacharjee S, Goldstone L, Vadiei N, Lee JK, Burke WJ. Depression screening patterns, predictors, and trends among adults without a depression diagnosis in ambulatory settings in the United States. Psychiatr Serv 2018;69(10):1098-1100. doi:https://doi.org/10.1176/ appi.ps.201700439.

28. Pignone MP, Gaynes BN, Rushton JL, et al. Screening for depression: systematic evidence review. 2002.

29. Forman-Hoffman V, McClure E, McKeeman J, et al. Screening for Major Depressive Disorder Among Children and Adolescents: A Systematic Review for the U.S. Preventive Services Task Force. Rockville: Agency for Healthcare Research and Quality; 2016.

30. Whooley MA. Screening for depression - a tale of two questions. JAMA Intern Med 2016. doi:https://doi.org/10.1001/jamainternmed.2015. 8493.

31. Richardson LP, Rockhill C, Russo JE, et al. Evaluation of the PHQ-2 as a brief screen for detecting major depression among adolescents. 
Pediatrics 2010;125(5):e1097-103. doi:https://doi.org/10.1542/peds. 2009-2712.

32. Goldberg $\mathbf{D}$. The value of screening in patient populations with high prevalence of a disorder. 2011. doi: https://doi.org/10.1186/2046-16824-13.

33. Kwan BM, Nease, DE. The State of the Evidence for Integrated Behavioral Health in Primary Care. In: Talen M, Burke Valeras A, eds. Integrated Behavioral Health in Primary Care. New York: Springer; 2013:65-98. doi:https://doi.org/10.1007/978-1-4614-6889-9_5.

34. Akincigil A, Olfson M, Siegel M, Zurlo KA, Walkup JT, Crystal S. Racial and ethnic disparities in depression care in community-dwelling elderly in the United States. Am J Public Health 2012;102:319-328. doi:https://doi.org/10.2105/AJPH.2011.300349.

35. Bauer MS, Leader D, Un H, Lai Z, Kilbourne AM. Primary care and behavioral health practice size: the challenge for health care reform. Med Care 2012;50(10):843-848. doi:https://doi.org/10.1097/MLR. Ob013e31825f2864.
36. Trangle M, Gursky J, Haight R, et al. Health care guideline: depression in primary care. 2016.

37. Gaynes BN, Rush AJ, Trivedi MH, et al. Primary versus specialty care outcomes for depressed outpatients managed with measurement-based care: results from STAR*D. J Gen Intern Med 2008;23(5):551-560. doi:https://doi.org/10.1007/s11606-008-0522-3.

Publisher's Note Springer Nature remains neutral with regard to jurisdictional claims in published maps and institutional affiliations. 